\title{
Non-Contrast-Enhanced Carotid MRA: Clinical Evaluation of a Novel Ungated Radial Quiescent-Interval Slice-Selective MRA at $1.5 \mathrm{~T}$
}

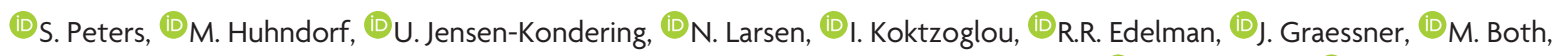
(1) O. Jansen, and $\mathbb{1}_{\mathrm{M}}$. Salehi Ravesh

\begin{abstract}
BACKGROUND AND PURPOSE: Non-contrast-enhanced MRA techniques have experienced a renaissance due to the known correlation between the use of gadolinium-based contrast agents and the development of nephrogenic systemic fibrosis and the deposition of gadolinium in some brain regions. The purpose of this study was to assess the diagnostic performance of ungated non-contrast-enhanced radial quiescent-interval slice-selective MRA of the extracranial supra-aortic arteries in comparison with conventional contrast-enhanced MRA in patients with clinical suspicion of carotid stenosis.
\end{abstract}

MATERIALS AND METHODS: In this prospective study, both MRA pulse sequences were performed in 31 consecutive patients (median age, 68.8 years; 19 men). For the evaluation, the cervical arterial system was divided into 35 segments (right and left side). Three blinded reviewers separately evaluated these segments. An ordinal scoring system was used to assess the image quality of arterial segments and the stenosis grading of carotid arteries.

RESULTS: Overall venous contamination in quiescent-interval slice-selective MRA was rated as "none" by all readers in $84.9 \%$ of cases and in $8.1 \%$ of cases in contrast-enhanced MRA $(P<.0001)$. The visualization quality of arterial segments was considered good to excellent in $40.2 \%$ for the quiescent-interval slice-selective MRA and in $52.2 \%$ for the contrast-enhanced MRA $(P<.0001)$. The diagnostic accuracy of ungated quiescent-interval slice-selective MRA concerning the stenosis grading showed a total sensitivity and specificity of $85.7 \%$ and $90.0 \%$, respectively.

CONCLUSIONS: Ungated quiescent-interval slice-selective MRA can be used clinically as an alternative to contrast-enhanced MRA without a significantly different image quality or diagnostic accuracy for the detection of carotid stenosis at 1.5T.

ABBREVIATIONS: $C E$ = contrast-enhanced; QISS = quiescent-interval slice-selective; $R F$ = radiofrequency; $C T A=$ computed tomography angiography; $D S A=$ digital subtracted angiography; DUS = duplex ultrasound; ECG = electro-cardiogram; FLASH = fast low angle shot; FOCI = frequency offset corrected inversion; MRA = magnetic resonance angiography; NASCET = North American Symptomatic Carotid Endarterectomy Trial

E xtracranial ICA stenosis is a common disease and a risk factor for an ischemic stroke. ${ }^{1}$ Atherosclerosis is the main cause of ICA stenosis. ${ }^{2,3}$ Typical risk factors for atherosclerosis are hypertension, history of smoking, diabetes, obesity, and an elevated low-density cholesterol level. ${ }^{4,5}$ An ICA stenosis can be treated either conservatively, for example with risk-factor control and best medical therapy, or invasively (endarterec-

Received April 1, 2019; accepted after revision July 4.

From the Department of Radiology and Neuroradiology (S.P., M.H., U.J.-K., N.L., M.B., O.J., M.S.R.), University Medical Center Schleswig-Holstein, Kiel University, Kiel, Germany; Department of Radiology (I.K., R.R.E.), NorthShore University Health System, Evanston, Illinois; University of Chicago Pritzker School of Medicine (I.K.), Chicago, Illinois; Northwestern University Feinberg School of Medicine (R.R.E.), Chicago, Illinois; and Siemens Healthcare (J.G.), Hamburg, Germany.

This work was supported, in part, by the National Institute of Biomedical Imaging and Bioengineering of the National Institutes of Health under award number R01EB027475.

The content is solely the responsibility of the authors and does not necessarily represent the official views of the National Institutes of Health. tomy or stent and angioplasty). ${ }^{3,6}$ The decision on the preferred method for the treatment of an ICA stenosis depends on several factors. Besides the existence of symptoms, the grade of the stenosis is an important factor. ${ }^{3,7}$ Therefore, determination of the stenosis grade is essential to assess the appropriate treatment.

Duplex sonography, CT angiography, and MR angiography are the non-minimally invasive imaging modalities to evaluate the ICA. ${ }^{8}$ The accuracy of a duplex ultrasound examination of the carotid arteries depends on the experience of the investigator, so a second imaging technique is required in most cases.

Please address correspondence to Mona Salehi Ravesh, MD, Department of Radiology and Neuroradiology, University Medical Center Schleswig-Holstein, Kiel University, Arnold Heller St 3, Bldg 41, 24105 Kiel, Germany; e-mail: Mona.Salehiravesh@uksh.de

- Indicates open access to non-subscribers at www.ajnr.org

三 Indicates article with supplemental on-line appendix.

http://dx.doi.org/10.3174/ajnr.A6171 


\begin{tabular}{|c|c|c|}
\hline Parameter & Ungated QISS-MRA & CE-MRA \\
\hline Imaging mode & $2 \mathrm{D}$ & $3 \mathrm{D}$ \\
\hline FLASH TR/TE (ms) & $15.0 / 4.7$ & $3.09 / 1.2$ \\
\hline QISS sequence TR (ms) & 1100.8 & - \\
\hline Acquisition matrix (Px) & $384 \times 384$ & $512 \times 512$ \\
\hline Acquisition pixel (mm²) & $0.5 \times 0.5$ & $0.6 \times 0.6$ \\
\hline In-plane interpolation & On & On \\
\hline Slice thickness (mm) & 2.0 & 1.0 \\
\hline No. of slices & 128 & 80 \\
\hline Slice distance factor (\%) & -33 & 20 \\
\hline No. of averages & 1 & 1 \\
\hline Receiver bandwidth (Hz/Px) & 303 & 540 \\
\hline Flip angle & $30^{\circ}$ & $30^{\circ}$ \\
\hline Slice orientation & Tilted transversal to coronal $\left(45^{\circ}\right.$ tilt $)$ & Coronal \\
\hline$K$-space trajectory & Radial & Cartesian \\
\hline No. of radial projections & 204 & - \\
\hline No. of shots per slice & 3 & - \\
\hline Phase oversampling (\%) & 0 & 40 \\
\hline Filter & $\begin{array}{l}\text { Distortion correction } \\
\text { (2D); prescan normalizer }\end{array}$ & $\begin{array}{l}\text { Distortion correction } \\
\text { (3D); prescan normalizer }\end{array}$ \\
\hline $\mathrm{B}_{0}$ shim mode & Heart & Tune-up \\
\hline Asymmetric echo & Off & On \\
\hline RF pulse type & Normal & Normal \\
\hline Gradient mode & Fast & Fast \\
\hline RF spoiler & On & On \\
\hline iPAT modus (acceleration factor/No. of reference lines) & - & $2 / 24$ \\
\hline Partial Fourier (phase and slice) & - & 6th/8th \\
\hline Venous saturation slab thickness (mm) & 100 & - \\
\hline Distance between venous saturation and imaging slab (mm) & 10 & - \\
\hline $\mathrm{TI}(\mathrm{ms})$ & 530 & - \\
\hline Acquisition time (min:sec) & 7:03 & $0: 20$ \\
\hline
\end{tabular}

Note:-iPAT indicates integrated parallel imaging technique; TI, time from in-plane and venous saturation to the acquisition of central $k$-space $\left(k_{\mathrm{y}}=0\right)$; Px, pixel; -, sequence parameter is not available; TR, repetition time; TE, echo time; RF, radiofrequency; $\mathrm{Hz}$, Hertz.

Additionally, extensive calcified plaques of the vessel wall impair accurate assessment of the grade of stenosis, which is difficult due to acoustic shadowing. Contrast-enhanced MRA (CE-MRA) using a gadolinium-based contrast agent is an often used minimally invasive method for grading an ICA stenosis. In patients with renal insufficiency, a gadolinium-based contrast agent should be used with caution. ${ }^{9}$ For these patients, the CE-CTA is not a suitable alternative due to the use of a potentially nephrotoxic iodine contrast medium. Furthermore, the deposition of a gadolinium-based contrast agent in the brain of patients even with good renal function is a subject of ongoing discussions and investigations into using non-CE MR imaging techniques. ${ }^{10,11}$ The above concerns about MR imaging contrast agent safety have spurred new developments in non-CE MR imaging techniques with reliable clinical results. ${ }^{12-14} 2 \mathrm{D} /$ 3D-TOF-MRA is a commonly used non-contrast-enhanced approach for MRA of the extracranial carotid arteries. However, both techniques are time-consuming compared with CEMRA. The image quality and anatomic coverage provided by TOF-MRA is inferior to that of CE-MRA. ${ }^{15,16}$ Moreover, TOF is more sensitive to respiratory and flow artifacts and has a tendency to overestimate stenoses. ${ }^{14}$

Recently, a new technique for non-CE-MRA of the arteries was presented. The so-called quiescent-interval slice-selective (QISS $)^{17}$ MRA was first used to examine the peripheral arteries and showed promising results. ${ }^{13}$ Recent advances in the QISS technique facilitate non-CE-MRA of other vessels, ${ }^{18}$ in particular the extracranial carotid arteries at 3T. ${ }^{19,20}$ The aim of this study was to test the feasibility of an ungated radial implementation of QISS-MRA at 1.5T and to assess its diagnostic performance for imaging the extracranial carotid arteries compared with the clinical standard technique of CE-MRA. For simplification, the ungated non-CE radial QISS-MRA is abbreviated as ungated QISS-MRA.

\section{MATERIALS AND METHODS Patients}

Patients who were included in this prospective study had been consecutively referred to our center from May to September 2018 for clinically indicated extracranial MRA of the supraaortic arteries. The medical history of all patients was reviewed to determine the reason for the clinically requested MR imaging examination of the carotid arteries. The study exclusion criteria were a history of carotid stent placement; renal insufficiency that precluded the administration of a gadoliniumbased contrast agent, as indicated by an estimated glomerular filtration rate of $<30 \mathrm{~mL} / \mathrm{min} / 1.73 \mathrm{~m}^{21}$; other contraindications for gadolinium-based contrast agent; and contraindications for MR imaging.

This study was performed according to the protocol (No. D 508/18) approved by the ethics committee at the university medical center in Kiel in accordance with the ethical standards established in the 1964 Declaration of Helsinki and its later amendments. Our patients gave written informed consent. 
Table 2: Evaluation of ungated QISS-MRA versus CE-MRA based on the introduced 3-, 4-, and 5-point scale scoring systems in the section "Image Analysis" using the Wilcoxon signed rank test

\begin{tabular}{|c|c|c|c|}
\hline Variable $^{a}$ & QISS-MRA ${ }^{b}$ & CE-MRA ${ }^{\mathrm{b}}$ & $\begin{array}{c}P \text { Value } \\
\text { (QISS-MRA vs } \\
\text { CE-MRA) }\end{array}$ \\
\hline Image quality & $2(1-3)$ & $2(1-3)$ & .46 \\
\hline Venous contamination & $0(0-2)$ & $1(0-3)$ & $<.0001$ \\
\hline Global quality of arterial visualization & $2(1-4)$ & $3(1-4)$ & $<.0001$ \\
\hline \multicolumn{4}{|l|}{ Stenosis grading } \\
\hline Right & $1(1-5)$ & $1(1-5)$ & .64 \\
\hline Left & $1(1-5)$ & $1(1-5)$ & .73 \\
\hline \multicolumn{4}{|l|}{ Segmental quality of arterial visualization } \\
\hline \multicolumn{4}{|l|}{ Right side } \\
\hline Origin of brachiocephalic artery (1) & $3(1-4)$ & $3(1-4)$ & $<.0001$ \\
\hline Origin of CCA (2) & $3(1-4)$ & $3(1-4)$ & $<.0001$ \\
\hline $\operatorname{CCA}(3)$ & $3(1-4)$ & $4(1-4)$ & .03 \\
\hline Bifurcation of CCA (4) & $3(1-4)$ & $4(1-4)$ & .002 \\
\hline $\mathrm{ICA}-\mathrm{Cl}$ (cervical) (5) & $3(1-4)$ & $4(1-4)$ & .011 \\
\hline ECA (superior thyroid artery) (6) & $1(1-3)$ & $2(1-4)$ & .007 \\
\hline ECA (lingual artery) (7) & $1(1-3)$ & $2(1-4)$ & .0002 \\
\hline ECA (facial artery) (8) & $2(1-3)$ & $2(1-4)$ & .0003 \\
\hline ECA (occipital artery) (9) & $2(1-3)$ & $2(1-4)$ & .043 \\
\hline ECA (posterior auricular artery) (10) & $1(1-3)$ & $1(1-4)$ & .16 \\
\hline ECA (suprafacial temporal artery) (11) & $2(1-3)$ & $2(1-4)$ & .002 \\
\hline ECA (maxillary artery) (12) & $2(1-3)$ & $2(1-4)$ & .001 \\
\hline ECA (ascending pharyngeal artery) (13) & $1(1-3)$ & $1(1-4)$ & .39 \\
\hline Origin of subclavian artery (14) & $2(1-4)$ & $3(1-4)$ & $<.0001$ \\
\hline Origin of vertebral artery (V0) (15) & $2(1-4)$ & $2(1-4)$ & .19 \\
\hline V1 (preforaminal) (16) & $3(1-4)$ & $3(1-4)$ & .064 \\
\hline V2 (foraminal) (17) & $3(1-4)$ & $3(1-4)$ & .51 \\
\hline V3 (atlantic, extradural, or extraspinal) (18) & $3(1-4)$ & $3(1-4)$ & .097 \\
\hline \multicolumn{4}{|l|}{ Left side } \\
\hline Origin of CCA (1) & $2(1-4)$ & $3(1-4)$ & .0003 \\
\hline CCA (2) & $3(2-4)$ & $4(1-4)$ & .01 \\
\hline Bifurcation of CCA (3) & $3(2-4)$ & $4(1-4)$ & .008 \\
\hline $\mathrm{ICA}-\mathrm{Cl}$ (cervical) (4) & $3(1-4)$ & $4(1-4)$ & .02 \\
\hline ECA (superior thyroid artery) (5) & $2(1-3)$ & $2(1-3)$ & .02 \\
\hline ECA (lingual artery) (6) & $1(1-3)$ & $2(1-3)$ & .002 \\
\hline ECA (facial artery) (7) & $2(1-3)$ & $2(1-4)$ & $<.0001$ \\
\hline ECA (occipital artery) (8) & $2(1-4)$ & $2(1-3)$ & .34 \\
\hline ECA (posterior auricular artery) (9) & $1(1-3)$ & $1(1-3)$ & .98 \\
\hline ECA (suprafacial temporal artery) (10) & $2(1-3)$ & $2(1-4)$ & .0003 \\
\hline ECA (maxillary artery) (11) & $2(1-3)$ & $2(1-4)$ & .0008 \\
\hline ECA (ascending pharyngeal artery) (12) & $1(1-3)$ & $1(1-3)$ & .34 \\
\hline Origin of subclavian artery (13) & $2(1-4)$ & $3(1-4)$ & $<.0001$ \\
\hline Origin of vertebral artery (V0) (14) & $2(1-4)$ & $2(1-4)$ & .001 \\
\hline V1 (preforaminal) (15) & $3(1-4)$ & $3(1-4)$ & .002 \\
\hline V2 (foraminal) (16) & $3(1-4)$ & $3(1-4)$ & .88 \\
\hline V3 (atlantic, extradural, or extraspinal) (17) & $3(1-4)$ & $3(1-4)$ & .11 \\
\hline
\end{tabular}

Note:-CCA indicates common carotid artery; ECA, external carotid artery.

${ }^{a}$ Numbers in parentheses indicate the arterial segments on the right and left sides.

${ }^{b}$ Data are median (minimum-maximum).

\section{Demographic Data of Study Population}

Pertinent demographic data (age, weight, body mass index at MR imaging, examination date, and sex) of the study population were recorded.

\section{MRA Imaging}

Imaging was performed on a $1.5 \mathrm{~T}$ MR imaging system (MAGNETOM Aera, XQ gradients, Siemens Healthcare GmbH, Erlangen, Germany) with a maximum gradient strength of 45 $\mathrm{mT} / \mathrm{m}$ and a maximum slew rate of $200 \mathrm{mT} / \mathrm{m} / \mathrm{ms}$. The MR imaging system was operated by the latest software (Syngo, version
E11C, Siemens Healthcare GmbH, Erlangen, Germany). The MR signal was received using a 20 -element head coil, a 4-element neck coil, and a 32-element array coil placed on the upper chest (Siemens Healthcare $\mathrm{GmbH}$, Erlangen, Germany)

The ungated QISS-MRA was performed in all subjects without electrocardiogram gating using a $2 \mathrm{D}$ singleshot radial FLASH readout. Flow compensation minimized blood flow artifacts.

Breath-hold first-pass CE-MRA was performed after ungated QISSMRA in all subjects with the administration of $0.1 \mathrm{mmol} / \mathrm{kg}$ body weight of gadolinium-based contrast agent (Gadovist, $1.0 \mathrm{mmol} / \mathrm{mL}$, gadobutrol; Bayer Pharma AG, Berlin, Germany) in an antecubital vein at a rate of 2 $\mathrm{mL} / \mathrm{s}$. After the CE-MRA examination, the CE-MRA images were subtracted from a native MRA image (mask), which was acquired with the same parameters before contrast agent injection. The imaging parameters for both MRA pulse sequences are summarized in Table 1.

\section{Image Analysis}

Three blinded board-certified radiologists (S.P., M.H., U.J.-K.) each with at least 7 years of experience in neuroradiology and MRA, evaluated all MR imaging datasets independently and during separate reading sessions. Source images and rotating MIP images were reviewed. The image analysis was performed on an Impax EE workstation (Agfa-Gevaert, Mortsel, Belgium).

The overall diagnostic quality of MR images was rated using a scoring scale of 1-3 with respect to the arterial signal and the presence of artifacts (including parallel acquisition reconstruction artifacts,

motion artifacts, and/or noise):

Grade 1: poor image quality, inadequate arterial signal, and/or the presence of a significant amount of artifacts/noise impairing the diagnosis.

Grade 2: good image quality sufficient for diagnosis, adequate arterial signal, and/or mild-to-moderate amounts of artifacts/noise not interfering with diagnosis.

Grade 3: Excellent image quality for highly confident diagnosis, good arterial signal, and no-to-minimal amount of artifacts/noise. 
Potentially contaminating venous signal was evaluated on a scale of $0-3$ :

Grade 0: none.

Grade 1: minimal, allowing interpretation with a high degree of diagnostic confidence.

Grade 2: moderate, exceeding acceptable degree and limiting diagnostic confidence.

Grade 3: severe, markedly limiting diagnostic confidence.

The cervical arteries were divided into 35 segments (Table 2).

The continuity, visibility, and edge sharpness of these segments were assessed in all subjects. Visualization of each segment was assessed using a scoring scale of 1-4:

Grade 1: nondiagnostic, barely visible lumen rendering of the segment.

Grade 2: fair, ill-defined vessel borders with suboptimal image quality for diagnosis.

Grade 3: good, minor inhomogeneities not influencing vessel delineation.

Grade 4: excellent, sharply defined arterial borders with excellent image quality for highly confident diagnosis.

The image quality of an arterial segment was deemed diagnostic (grade $\geq 3$ ) if the reader was confidently able to visualize the lumen of the structure of the carotid artery in its entirety.

The stenosis grading of the right and left internal carotid arteries was evaluated on the basis of the NASCET trial ${ }^{22}$ criteria using a scoring scale of 1-5:

Grade 1: 0\% normal patency.

Grade 2: $<50 \%$ stenosis.

Grade 3: 50\%-69\% stenosis.

Grade 4: $\geq 70 \%$ stenosis.

Grade 5: 100\% occlusion.

Table 3: Interobserver agreement for the evaluation of QISSMRA and CE-MRA based on the introduced 3-, 4-, and 5-point scale scoring systems in the "Image Analysis" section ${ }^{\mathrm{a}}$

\begin{tabular}{lc}
\multicolumn{1}{c}{ Variable } & $\begin{array}{c}\text { Interobserver } \\
\text { Agreement }\end{array}$ \\
\hline Image quality & $0.54(0.46-0.62)$ \\
Venous contamination & $0.86(0.80-0.91)$ \\
Quality of global arterial visualization & \\
$\quad$ Right side & $0.72(0.70-0.74)$ \\
Left side & $0.71(0.69-0.72)$ \\
ICA stenosis & \\
$\quad$ Right side & $0.94(0.89-0.97)$ \\
Left side & $0.95(0.90-0.98)$ \\
\hline
\end{tabular}

When multiple stenotic lesions occurred in a particular arterial segment, the most stenotic lesion was considered the diagnostic grade and was used in the analysis.

Detailed information about the ungated QISS-MRA and the statistical analysis are available in the On-line Appendix.

\section{RESULTS}

\section{Patient Characteristics}

Our study population consisted of 31 patients (median age, 65.0 years [range, 27.7-91.4 years]; weight, $79.2 \mathrm{~kg}$ [range, 53.4-120.0 $\mathrm{kg}$ ]; body mass index, $26.3 \mathrm{~kg} / \mathrm{m}^{2}$ [range, $17.4-37.9 \mathrm{~kg} / \mathrm{m}^{2}$ ]), including 19 male and 12 female subjects. In 21 subjects, the MR imaging was requested due to the suspicion of an arterio-arterial embolic ischemic stroke or a suspected stroke, in 5 cases for exclusion of a severe carotid stenosis before a cardiac or aortic operation, and in 5 cases to exclude a dissection of the cervical arteries after a trauma or after previous dissections.

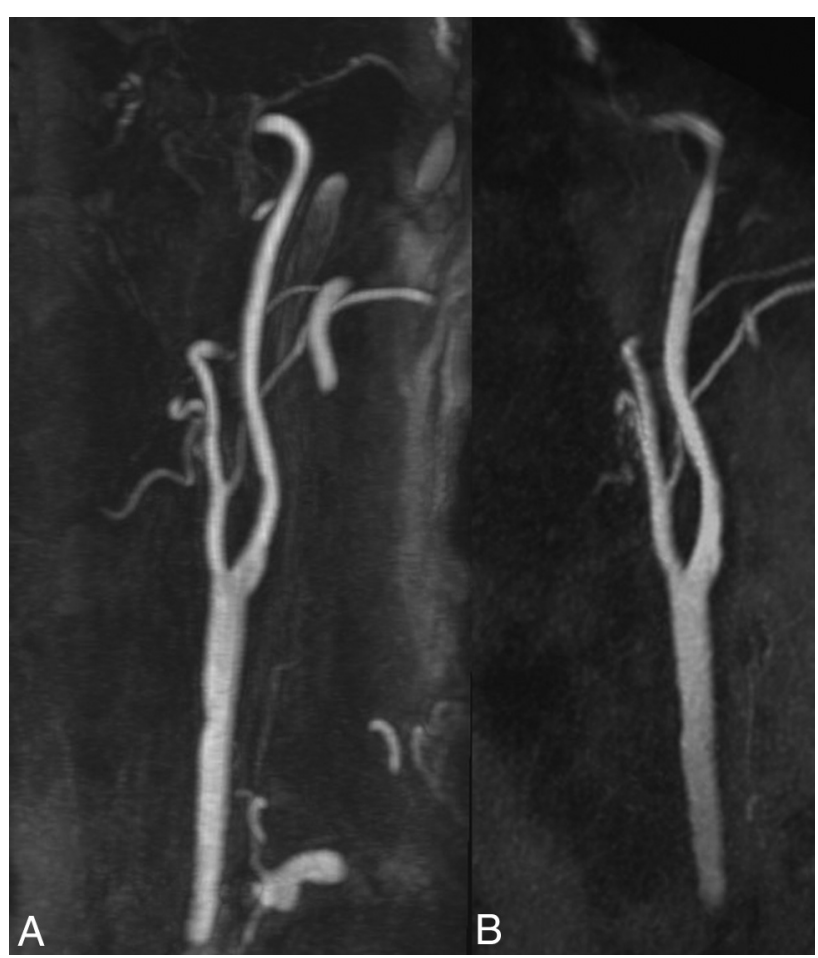

FIG 1. Example of an excellent imaging quality (grade 3) without any venous contamination (grade 0). Maximum intensity projection (MIP) with angulation to the left carotid bifurcation of the CE-MRA ( $A$, slice thickness: $14.5 \mathrm{~mm}$ ) and the ungated QISS-MRA ( $B$, slice thickness: 14.1 $\mathrm{mm}$ ) of a 76-year-old patient with clinically suspected infarction of the right hemisphere and suspected stenosis of the right cervical internal carotid artery by sonography (same patient as in Fig 5).

Table 4: Comparison of ungated QISS-MRA and CE-MRA for assessment of the stenosis grade of the extracranial carotid arteries ${ }^{\mathrm{a}}$

\begin{tabular}{|c|c|c|c|c|c|c|}
\hline & \multicolumn{3}{|c|}{ Right Side $^{\mathrm{b}}$} & \multicolumn{3}{|c|}{ Left Side $^{b}$} \\
\hline & 1 & 2 & 3 & 1 & 2 & 3 \\
\hline Sensitivity (\%) & $66.7(9.4-99.2)$ & $100.0(15.8-100.0)$ & $50.0(1.3-98.7)$ & $100(39.8-100.0)$ & $83.3(35.9-99.6)$ & $100.0(39.8-100.0)$ \\
\hline All readers & & $71.4(29.0-96.3)$ & & & 92.9 (66.1-99.8) & \\
\hline Both sides & \multicolumn{6}{|c|}{$85.7(63.7-97.0)$} \\
\hline Specificity (\%) & $89.3(71.8-97.7)$ & $86.2(68.3-96.1)$ & 89.7 (72.7-97.8) & $92.6(75.7-99.1)$ & $88.0(68.8-97.5)$ & $96.3(81.0-99.9)$ \\
\hline All readers & & $87.7(78.5-93.3)$ & & & $92.4(84.2-97.2)$ & \\
\hline Both sides & \multicolumn{6}{|c|}{$90.0(84.3-94.2)$} \\
\hline
\end{tabular}

\footnotetext{
${ }^{a}$ Data are sensitivity/specificity $(95 \% \mathrm{Cl})$.
}

${ }^{\mathrm{b}}$ Numbers 1, 2, and 3 refer to the readers. 


\section{Image Quality}

A total of 62 datasets (31 datasets per QISS-MRA and CE-MRA) were evaluated by 3 readers.

For QISS-MRA, reader 1 graded the overall image quality in 6.5\% (2/31) as "poor," in 38.7\% (12/31) as "good," and in 54.8\% (17/31) of cases as "excellent"; reader 2 graded $16.1 \%(5 / 31)$ as poor, $71.0 \%$ $(22 / 31)$ as good, and $12.9 \%(4 / 31)$ of cases as excellent; and reader 3 graded 3.2\% (1/31) as poor, $93.5 \%(29 / 31)$ as good, and 3.2\% (1/31) of cases as excellent.

For CE-MRA, reader 1 graded the overall image quality in $9.7 \%(3 / 31)$ as poor, in $48.4 \%(15 / 31)$ as good, and in $41.9 \%$ (13/31) of cases as excellent; reader 2 graded $16.1 \%(5 / 31)$ as poor, $38.7 \%(12 / 31)$ as good, and $45.2 \%(14 / 31)$ of cases as excellent; and reader 3 graded $9.7 \%(3 / 31)$ as poor, $80.6 \%(25 / 31)$ as good, and $9.7 \%(3 / 31)$ of cases as excellent.

Image quality was graded in 23.7\% (22/93) of QISS-MRA cases and in $32.3 \%$ (27/93) of CE-MRA cases as excellent by all readers. There was no significant difference between the two MRA pulse sequences concerning the image quality ( 2 median [range, $1-3$ ] versus 2 median [range, $1-3$ ], $P=.46$, Table 2 ).

\section{Venous Contamination}

For QISS-MRA, reader 1 graded the contaminating venous signal in $77.4 \%(24 / 31)$ as "none," in 19.4\% (6/31) as "minimal," and in $3.2 \%(1 / 31)$ as "moderate"; reader 2 graded 93.5\% (29/31) as none, $3.2 \%(1 / 31)$ as minimal, and $3.2 \%(1 / 31)$ as moderate; and reader 3 graded $83.9 \%(26 / 31)$ as none and $16.1 \%(5 / 31)$ as minimal.

For CE-MRA, reader 1 graded the contaminating venous signal in $3.2 \%(1 / 31)$ as none, in $83.4 \%(26 / 31)$ as minimal, in $6.5 \%$ $(2 / 31)$ as moderate, and in $6.5 \%(2 / 31)$ of cases as "severe"; reader 2 graded $12.9 \%(4 / 31)$ as none, $64.5 \%(20 / 31)$ as minimal, $16.1 \%$ $(5 / 31)$ as moderate, and $6.5 \%(2 / 31)$ as severe; and reader 3 graded $9.7 \%(3 / 31)$ as none, $61.3 \%(19 / 31)$ as minimal, $19.4 \%$ $(6 / 31)$ as moderate, and $9.7 \%(3 / 31)$ of cases as severe.

Overall venous contamination was rated as none by all readers in $84.9 \%$ (79/93) of QISS-MRA cases and in $8.1 \%(8 /$ 93) of CE-MRA cases ( 0 [range, $0-2$ ] versus 1 [range, $0-3$ ], $P<$ .0001 , Table 2).

\section{Visualization of Arterial Segments}

A total of 3255 arterial segments (35 arterial segments, 18 on the right side and 17 on the left side) for each patient per QISS-MRA and CE-MRA were evaluated once by all 3 readers.

For QISS-MRA, reader 1 scored 11.2\% (121/1085) of segments with grade four, $30.9 \%$ (335/1085) with grade three, $29.8 \%$ with grade two (323/1085), and 28.2\% (306/1085) with grade 1. Reader 2 identified $11.2 \%$ (121/1085) of segments with grade four, $30.6 \%$ (332/1085) with grade three, $45.0 \%$ with grade two (488/1085), and $13.3 \%$ (144/1085) with grade 1 . Reader 3 graded $11.6 \%(126 / 1085)$ of segments with grade four, $25.2 \%(273 / 1085)$ with grade three, $30.5 \%$ with grade 2 (331/1085), and 32.7\% (355/ 1085) with grade 1 .

The overall median rating grades of all readers was 2 (range, 1-4).

For CE-MRA, reader 1 identified 22.3\% (242/1085) of segments with grade four, $28.1 \%$ (305/1085) with grade three, $28.6 \%$

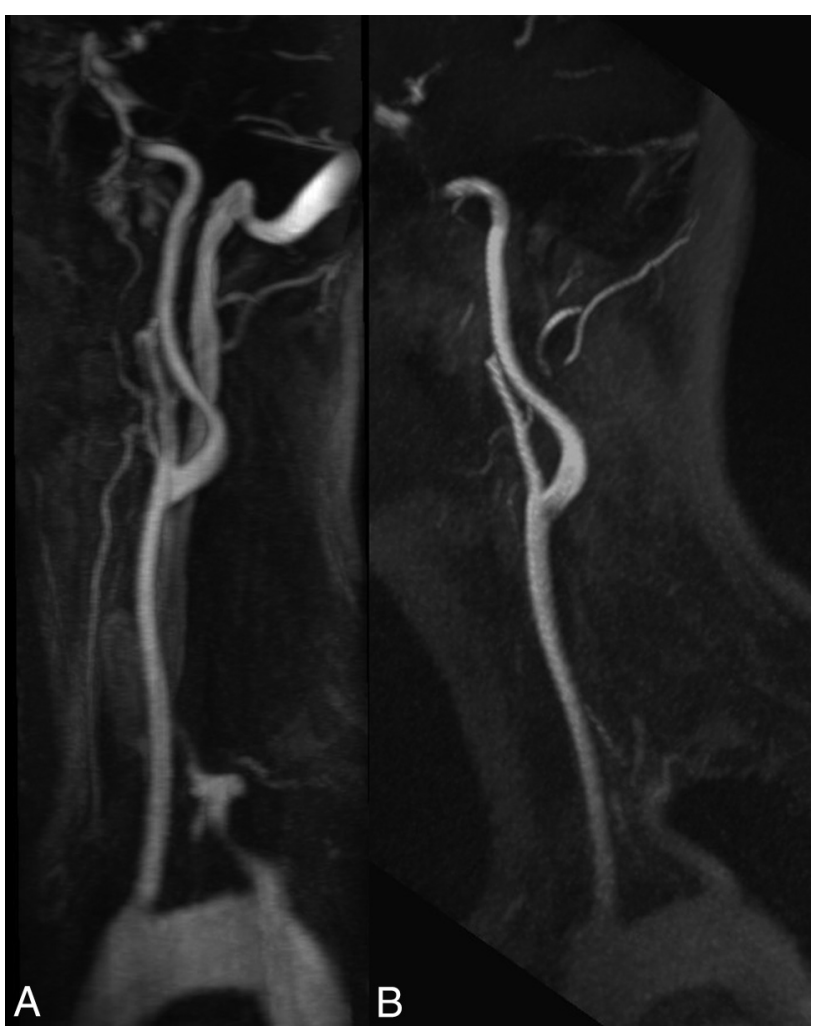

FIG 2. The effect of venous contamination on the image quality. MIP with angulation to the left carotid bifurcation of the CE-MRA ( $A$, slice thickness: $13.9 \mathrm{~mm}$ ) and the QISS-MRA ( $B$, slice thickness: $13.5 \mathrm{~mm}$ ) of a 33-year-old patient with suspected cerebral infarction. In the CEMRA, the bolus is slightly missed, resulting in a severe venous contamination, whereas the QISS-MRA shows no venous signal.

with grade two (310/1085), and 21.0\% (228/1085) with grade 1. Reader 2 scored $28.6 \%$ (310/1085) of segments with grade four, $36.3 \%$ (394/1085) with grade three, $29.0 \%$ with grade two (315/ $1085)$, and $6.1 \%(66 / 1085)$ with grade 1 . Reader 3 graded $16.8 \%$ (182/1085) of segments with grade four, $24.6 \%$ (267/1085) with grade three, $28.8 \%$ with grade two (312/1085), and 29.9\% (324/ 1085) with grade 1

The overall median rating grades of readers 1 and 2 was 3 (range, 1-4), and of reader 3 , it was 2 (range, 1-4).

The visualization quality of arterial segments was considered good to excellent (grade $\geq 3$ ) in $40.2 \%$ (1308/3255) for the QISSMRA and in 52.2\% (1700/3255) for the CE-MRA (2 [range, 1-4] versus 3 [range, $1-4$ ],$P<.0001)$. A detailed comparison among all arterial segments between both MRA-pulse sequences is shown in Table 2.

There was a strong correlation between the QISS-MRA and CE-MRA sequence concerning the detection of carotid stenosis on both sides $(r=0.92, P<.0001)$ with an excellent interobserver agreement of 0.94 for both sides (Table 3 ).

The interobserver agreement for the QISS-MRA and CE-MRA concerning the image quality was 0.54 , contamination with the venous enhancement was 0.86 , and visualization of arterial segments on the left and right sides was 0.71 .

Detailed information about the evaluation results are available in Tables 2, 3, and 4. Clinical examples are provided in Figs 1-5. 


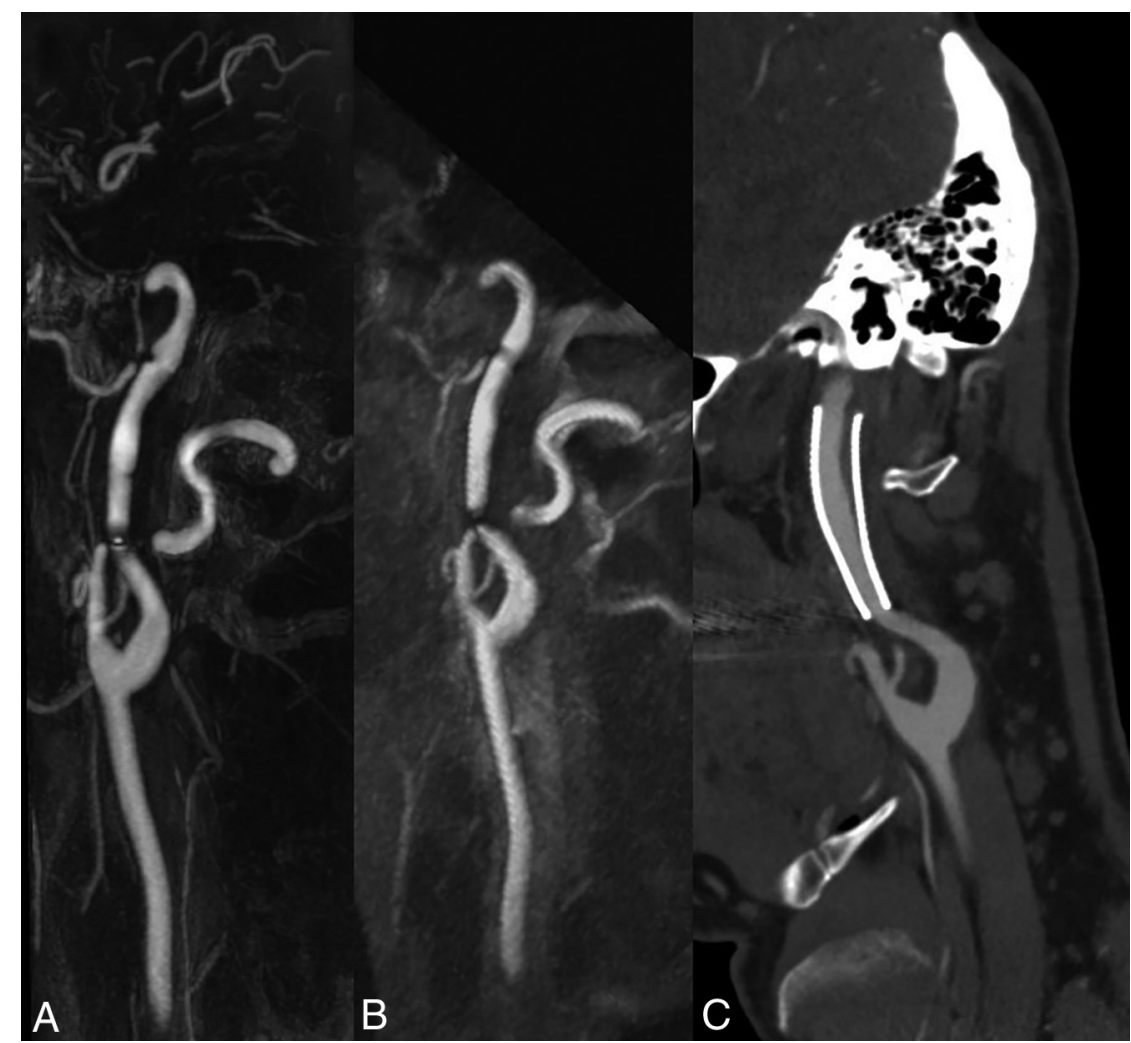

FIG 3. Influence of an implanted stent on the image quality. MIP of the CE-MRA (A, slice thickness: $13.0 \mathrm{~mm}$ ) and the ungated QISS-MRA ( $B$, slice thickness: $13.0 \mathrm{~mm}$ ) with angulation to the left internal carotid artery of a 50-year-old patient who was stented 5 years ago due to a carotid artery dissection. The corresponding MIP of a CE-CTA (C, slice thickness: $1.4 \mathrm{~mm}$ ) was obtained 2 years, and DSA, lyear after stent placement. In both MRA techniques, there are just slight artifacts at the ends of the stent, and the lumen is well visualized. This patient was not included in this study.

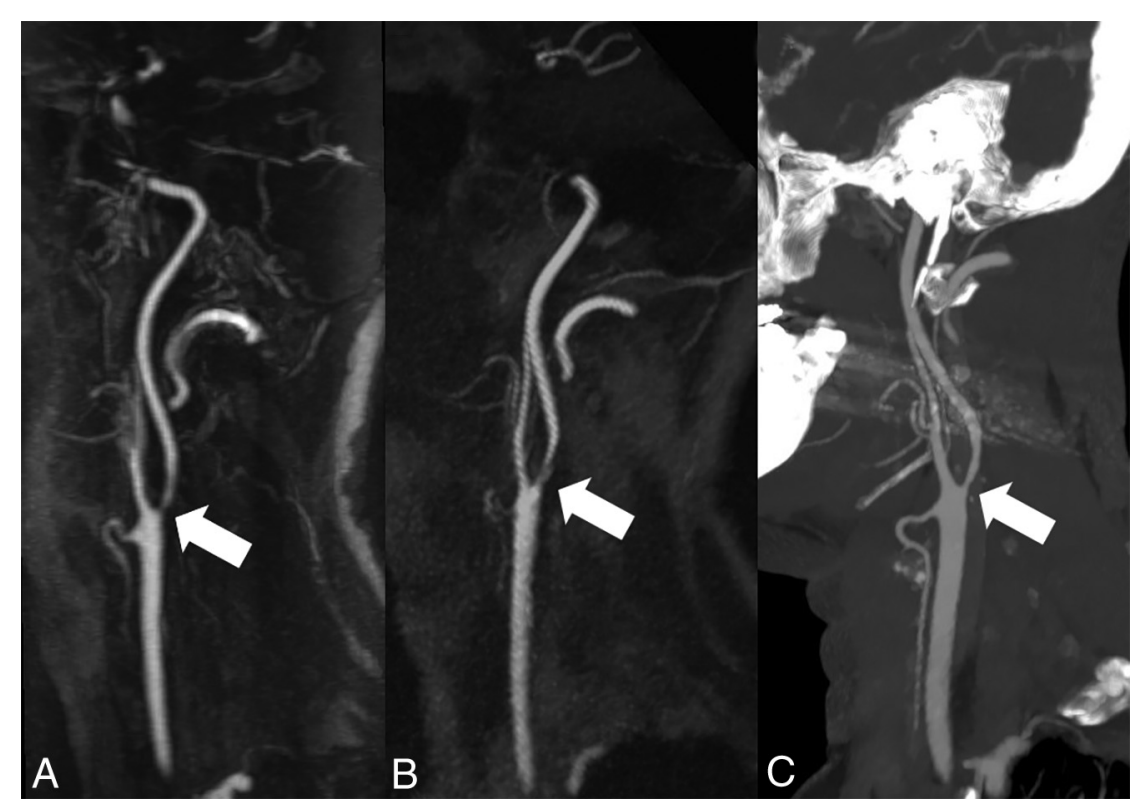

FIG 4. Visualization of internal carotid artery stenosis using CE-MRA and ungated QISS-MRA compared with CE-CTA. MIP with angulation to the left carotid bifurcation of the CE-MRA (A, slice thickness: $13.1 \mathrm{~mm}$ ), QISS-MRA ( $B$, slice thickness: $13.0 \mathrm{~mm}$ ), and CE-CTA (C, slice thickness: 13.0 $\mathrm{mm}$ ) of a 55-year-old patient with confirmed infarction of the left hemisphere and suspected stenosis of the left internal carotid artery using sonography. All 3 techniques verified the diagnosis of carotid stenosis (white arrows).

\section{DISCUSSION}

Due to the known correlation between the use of gadolinium-based contrast agents and the development of nephrogenic systemic fibrosis in patients with end-stage renal disease ${ }^{9}$ and also the deposition of gadolinium in some brain regions, ${ }^{10}$ non-CE-MRA techniques have experienced a renaissance in research and development and clinical application. ${ }^{12,23}$

Since the introduction of non-CE QISS-MRA for evaluating the lower extremities in 2010, ${ }^{17}$ this technique and its variants have been used and clinically evaluated in a variety of vascular territories. In 2016, Koktzoglou et $\mathrm{al}^{19}$ presented the feasibility of a cardiac-gated Cartesian QISS sequence variant for non-CE MRA of the extracranial carotid arteries in 5 healthy volunteers and 5 patients at 3T. The results of QISS were compared with those of 2D-TOF and CE-MRA, and they found that QISS provided better image quality than $2 \mathrm{D}$ TOF. Moreover, their initial results suggested that cardiac-gated QISS has potential utility as a non-CE alternative to CE-MRA. A recent retrospective study conducted at $3 \mathrm{~T}$ has also demonstrated improved image quality of radial QISS with respect to $2 \mathrm{D}-\mathrm{TOF}^{20}$

In our prospective study, for the first time the diagnostic accuracy of ungated QISS-MRA was compared with CEMRA in patients with suspected extracranial carotid artery stenosis at $1.5 \mathrm{~T}$. The main findings of this study are the following: 1) QISS-MRA provides good visualization of the supra-aortic arteries without contrast agent and without cardiac gating; 2) on the basis of the performed segmental evaluation by 3 experienced radiologists, ungated QISSMRA showed high sensitivity and specificity and a significant correlation with CE-MRA for the detection of carotid artery stenosis; and 3) ungated QISS-MRA is therefore a reliable technique for diagnosing carotid artery stenosis, in particular in patients with contraindications to gadolinium-based contrast agents.

QISS was originally described as a technique that leverages cardiac gating to optimally synchronize the quiescent intervals and readout to rapid systolic and slow diastolic arterial flow, respec- 


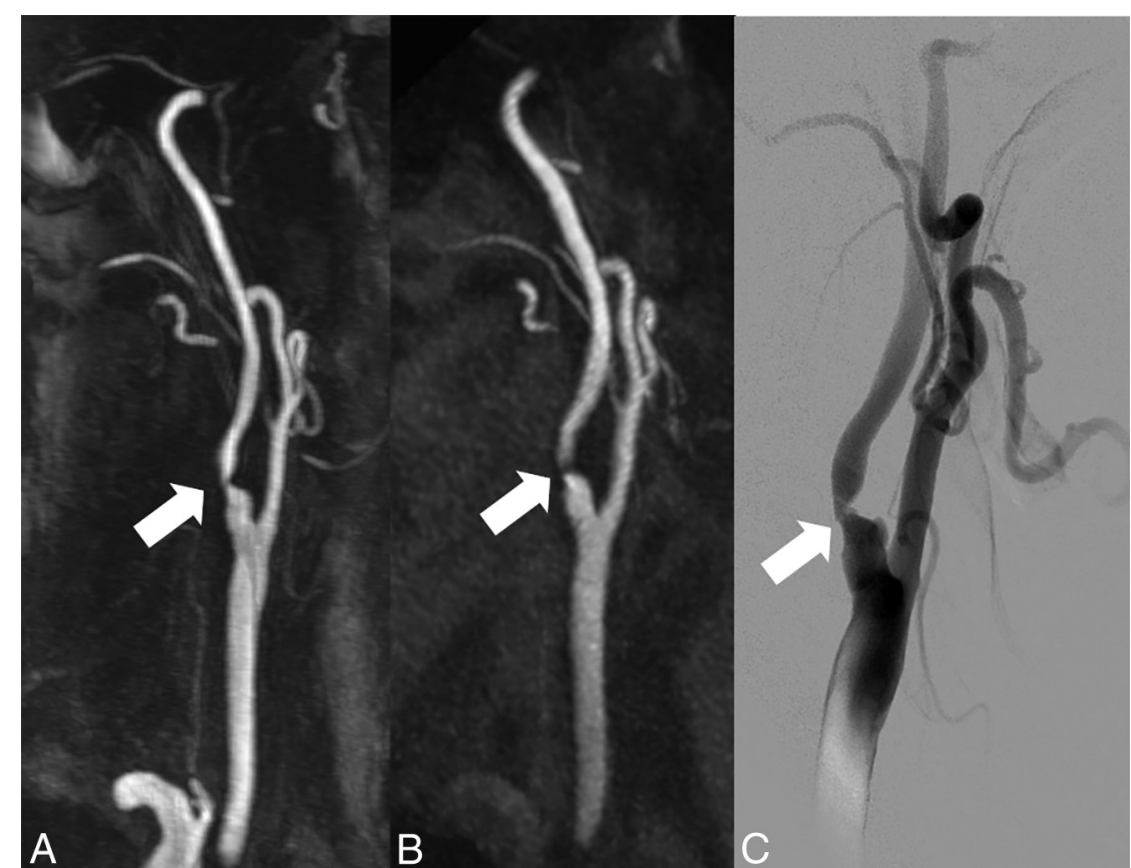

FIG 5. Visualization of internal carotid artery stenosis using CE-MRA and ungated QISS-MRA compared with invasive DSA. MIP with angulation to the right carotid bifurcation of the CE-MRA ( $A$, slice thickness: $14.0 \mathrm{~mm}$ ) and the QISS-MRA (B, slice thickness: $13.5 \mathrm{~mm}$ ) of a 76-year-old patient with clinically suspected infarction of the right hemisphere and suspected stenosis of the cervical internal carotid artery on the right by sonography (same patient as in Fig 1). The corresponding DSA of the right carotid bifurcation (C) before stent angioplasty confirmed the stenosis (white arrows).

tively. However, because most MR imaging protocols for imaging in the head and neck are performed without cardiac or peripheral pulse triggering, it is most convenient from a clinical perspective to image without cardiac synchronization.

In this study, an ungated implementation of the QISS-MRA pulse sequence leveraging radial $k$-space sampling was used. The consistent arterial contrast obtained using QISS-MRA in this study was predicated on 3 factors: first, the continuous flow found in the brain circulation; second, the combination of a rather lengthy interecho spacing $(\sim 15 \mathrm{~ms})$ and a low flip angle $\left(30^{\circ}\right)$, which minimized saturation of arterial flow; and third, the use of radial $k$-space sampling to suppress arterial pulsation artifacts.

The duration of the measurement time using ungated QISSMRA was fixed to 7 minutes and was independent of patient heart rate and electrocardiogram quality. While the measurement time of CE-MRA on paper is only 20 seconds, the total time to perform CE-MRA is, in fact, longer than that needed for ungated QISSMRA. This is due to the extra time required for the preparation of patients for contrast agent injection, acquisition of a precontrast dataset as a mask for CE-MRA ( $\sim 20$ seconds), and also the postprocessing of the CE dataset ( $\sim 30$ seconds), which is not needed for the ungated QISS-MRA procedure. The ungated QISS-MRA can be repeated as often as required without problems with venous contamination, for instance for the diagnosis of various arterial abnormalities in head and neck region and also for serial follow-up imaging. Images of the intracranial arteries can also be acquired in addition to the extracranial arteries when the slicedistance factor is set to a value of $-20 \%$ to $-25 \%$ instead of
$-33 \%$ (used in this study), without any extension of measurement time.

As demonstrated in this study, the image quality of ungated QISS-MRA was comparable with that of CE-MRA and was graded as good or excellent in most cases. In some cases, the informative value of the ungated QISS-MRA was even higher due to less venous signal. There was almost no residual venous signal observed in the ungated QISSMRA. This result indicates sufficient suppression of venous spins by the tracking venous inversion radiofrequency (RF) pulse, despite the use of tilted slices. Compared with axial slices, a possible drawback of tilted slices is the potential for insufficient inflow into vessel segments parallel to the slice direction. A slight reduction of arterial signal intensity was most visible in the aortic arch near the aortic branches. On the basis of our data, however, this reduction of image contrast does not affect the diagnostic accuracy for grading a carotid stenosis. In comparison, some CE-MRA examinations showed severe venous contamination due to mistiming of the image acquisition with respect to the first pass of the contrast bolus.

The acquisition of a CE-MRA dataset of the extracranial carotid arteries was performed in breath-hold to reduce the image artifacts due to respiratory motion. To avoid possible image artifacts during swallowing, we asked the patients to stop swallowing (eg, for about 20 seconds). In contrast, ungated QISS-MRA could be performed in free breathing. The ungated QISS-MRA pulse sequence is largely insensitive to respiratory motion and arterial pulsation artifacts due to its use of radial $k$-space sampling, which oversamples the center of the $k$-space and dilutes the impact of respiratory and flow-related signal fluctuations that occur in a minority of radial views. These physiological signal fluctuations were suppressed with the use of radial $k$-space sampling so far that there was no residual stripping apparent with ungated QISSMRA. Meanwhile, the use of an image-based navigator reduced the impact of intermittent swallowing motion artifacts.

All 35 extracranial segments with different shapes, lengths, and diameters were analyzed in our study to show even small, clinically irrelevant differences in imaging quality. The CE-MRA provided slightly better visualization of the small vessels. However, these differences did not influence patient management. Moreover, the clinically relevant findings were also reliably detected by the QISS-MRA.

The results of ungated QISS-MRA correlated strongly with those of CE-MRA concerning the stenosis grading. All 3 neuroradiologists graded the carotid artery stenosis in nearly $90 \%$ of seg- 
ments (right side, $87.0 \%$; left side, $93.5 \%$ ) with the same score in ungated QISS-MRA and CE-MRA. In 5 cases, QISS-MRA overestimated the grade of stenosis, and in 1 case, QISS-MRA underestimated it. In 2 cases, the overestimation and in 1 case the underestimation of stenosis grading by QISS-MRA led to a change between grade 1 and 2 ( $0 \%$ to $50 \%$ stenosis) without therapeutic relevance.

In 2 cases, the overestimation of stenosis grading by QISSMRA led to a change between grades 2 and 3. In one of these cases, the stenosis overestimation would have affected patient management due to the presence of symptoms for the right carotid stenosis, but in the other case, this overestimation did not affect the therapy management because the patient did not have the required symptoms on that side.

In the last case, the diagnosis based on the results of QISSMRA led to an overestimation of the stenosis grade in the right carotid artery from 3 to 4 . This change in grading did not affect the therapy management because therapy was indicated due to the symptoms on this side. Discrepancies in stenosis evaluation occur not only between QISS-MRA and CE-MRA but also among different modalities used for the assessment of ICA stenosis such as CE-CT, duplex ultrasound, and DSA. In cases with discrepant results or borderline stenosis grading, we perform conventional angiography prepared for optional stent implantation. Therefore, relevant stenosis will not be missed and will be treated. The diagnostic accuracy of ungated QISS-MRA showed a total sensitivity and specificity of $85.7 \%$ and $90.0 \%$, respectively. Furthermore, the evaluation of the ICA stenosis grading revealed an excellent interobserver agreement. These data indicate that ungated QISSMRA can be potentially used as an alternative to CE-MRA for grading carotid artery stenosis.

The number of patients in our single-center study was relatively small. Potentially, a higher number of patients in a multicenter study is necessary to confirm the diagnostic performance of ungated QISS-MRA across a wider range of clinical indications.

\section{CONCLUSIONS}

This study indicates that ungated QISS-MRA is a reliable angiographic technique with significant clinical potential for the visualization of the extracranial carotid arteries and detection of their stenosis at 1.5T. Ungated QISS-MRA is a feasible alternative for patients with contraindications to gadolinium-based contrast agents, especially in high-risk patients with severe renal insufficiency and an irregular cardiac rhythm. Furthermore, ungated QISS-MRA can avoid the timing-related difficulties of CE-MRA.

Disclosures: Sönke Peters-UNRELATED: Employment: University Hospital Schleswig-Holstein. Ulf Jensen-Kondering-UNRELATED: Grants/Grants Pending: grant from the Medical Faculty. loannis Koktzoglou—RELATED: Grant: National Institute of Biomedical Imaging and Bioengineering of the National Institutes of Health, Comments: Award No. R01EB027475*; UNRELATED: Other: Siemens, Comments: research support.* Robert R. Edelman—UNRELATED: Grants/Grants Pending: Siemens, Comments: Research support*; Royalties: Siemens, Comments: royalties relating to license for noncontrast MRA. Joachim Graessner-UNRELATED: Employment: Siemens. Olav Jansen-UNRELATED: Consultancy: Route 92 Medical; Payment for Lectures Including Service on Speakers Bureaus: Stryker, MicroVention, Bayer Pharma AG. *Money paid to the institution.

\section{REFERENCES}

1. Flaherty ML, Kissela B, Khoury JC, et al. Carotid artery stenosis as a cause of stroke. Neuroepidemiology 2013;40:36-41 CrossRef Medline

2. Boehme AK, Esenwa C, Elkind MS. Stroke risk factors, genetics, and prevention. Circ Res 2017;120:472-95 CrossRef Medline

3. Padalino DJ, Deshaies EM. Management of atherosclerotic carotid artery stenosis. In: Rezzani R, ed. Carotid Artery Disease: From Bench to Beside and Beyond. Rijeka: InTech; 2014

4. Hobson RW 2nd, Mackey WC, Ascher E, et al; Society for Vascular Surgery. Management of atherosclerotic carotid artery disease: clinical practice guidelines of the Society for Vascular Surgery. $J$ Vasc Surg 2008;48:480-86 CrossRef Medline

5. Fishbein MC, Fishbein GA. Arteriosclerosis: facts and fancy. Cardiovasc Pathol 2015;24:335-42 CrossRef Medline

6. Wabnitz AM, Turan TN. Symptomatic carotid artery stenosis: surgery, stenting, or medical therapy? Curr Treat Options Cardiovasc Med 2017;19:62 CrossRef Medline

7. Abbott AL, Paraskevas KI, Kakkos SK, et al. Systematic review of guidelines for the management of asymptomatic and symptomatic carotid stenosis. Stroke 2015;46:3288-301 CrossRef Medline

8. Adla T, Adlova R. Multimodality imaging of carotid stenosis. Int J Angiol 2015;24:179-84 CrossRef Medline

9. Grobner T. Gadolinium: a specific trigger for the development of nephrogenic fibrosing dermopathy and nephrogenic systemic fibrosis? Nephrol Dial Transplant 2006;21:1104-08 CrossRef Medline

10. Kanda $\mathrm{T}$, Ishii $\mathrm{K}$, Kawaguchi $\mathrm{H}$, et al. High signal intensity in the dentate nucleus and globus pallidus on unenhanced T1-weighted MR images: relationship with increasing cumulative dose of a gadolinium-based contrast material. Radiology 2014;270:834-41 CrossRef Medline

11. Costa AF, van der Pol CB, Maralani PJ, et al. Gadolinium Deposition in the Brain: A Systematic Review of Existing Guidelines and Policy Statement Issued by the Canadian Association of Radiologists. Can Assoc Radiol J 2018;69:373-82 CrossRef Medline

12. Kramer H, Runge VM, Morelli JN, et al. Magnetic resonance angiography of the carotid arteries: comparison of unenhanced and contrast enhanced techniques. Eur Radiol 2011;21:1667-76 CrossRef Medline

13. Amin P, Collins JD, Koktzoglou I, et al. Evaluating peripheral arterial disease with unenhanced quiescent-interval single-shot MR angiography at 3 T. AJR Am J Roentgenol 2014;202:886-93 CrossRef Medline

14. Weber J, Veith P, Jung B, et al. MR angiography at 3 Tesla to assess proximal internal carotid artery stenoses: contrast-enhanced or 3D time-of-flight MR angiography? Clin Neuroradiol 2015;25:414-18 CrossRef Medline

15. Yucel EK, Anderson CM, Edelman RR, et al. AHA scientific statement: magnetic resonance angiography-update on applications for extracranial arteries. Circulation 1999;100:2284-301 CrossRef Medline

16. Huston J 3rd, Fain SB, Riederer SJ, et al. Carotid arteries: maximizing arterial to venous contrast in fluoroscopically triggered contrast-enhanced MR angiography with elliptic centric view ordering. Radiology 1999;211:265-73 CrossRef Medline

17. Edelman RR, Sheehan JJ, Dunkle E, et al. Quiescent-interval single-shot unenhanced magnetic resonance angiography of peripheral vascular disease: technical considerations and clinical feasibility. Magn Reson Med 2010;63:951-58 CrossRef Medline

18. Edelman RR, Carr M, Koktzoglou I. Advances in non-contrast quiescent-interval slice-selective (QISS) magnetic resonance angiography. Clin Radiol 2019;74:29-36 CrossRef Medline

19. Koktzoglou I, Murphy IG, Giri S, et al. Quiescent interval low angle shot magnetic resonance angiography of the extracranial carotid arteries. Magn Reson Med 2016;75:20727-27 CrossRef Medline 
20. Koktzoglou I, Aherne EA, Walker MT, et al. Ungated nonenhanced radial quiescent interval slice-selective (QISS) magnetic resonance angiography of the neck: evaluation of image quality. J Magn Reson Imaging 2019 May 11. [Epub ahead of print] CrossRef Medline

21. ACR Committee on Drugs and Contrast Media. ACR Manual on Contrast Media. Version 10.3, 2018: https://www.acr.org/-/media/ACR/ Files/Clinical-Resources/Contrast_Media.pdf; 2018. Accessed March 10,2019
22. North American Symptomatic Carotid Endarterectomy Trial: methods, patient characteristics, and progress. Stroke 1991;22: 711-20 CrossRef Medline

23. Koktzoglou I, Walker MT, Meyer JR, et al. Nonenhanced hybridized arterial spin labeled magnetic resonance angiography of the extracranial carotid arteries using a fast low angle shot readout at 3 Tesla. J Cardiovasc Magn Reson 2016;18:18 CrossRef Medline 